\title{
Network Pharmacology of JinChaiKangBingDu Capsule Against Influenza A (H1N1) Virus Infection
}

\author{
Zhi-de CHENG ${ }^{1}$, Xiao-jing WANG ${ }^{1}$, Peng $\mathrm{DENG}^{1}$, Jing $\mathrm{ZHAO}^{2,3,{ }^{*}}$ \\ and Gao CHEN ${ }^{1, a, *}$ \\ ${ }^{1}$ School of Life Sciences, Hubei University, Wuhan, 430062, China \\ ${ }^{2}$ Institute of Basic Research in Clinical Medicine, China Academy of Traditional \\ Chinese Medicine, Beijing, 100700, China \\ ${ }^{3}$ Center for East-West Medicine, University of California, Los Angeles, USA \\ agc_npwr@126.com \\ ${ }^{*}$ Corresponding author
}

Keywords: JinChaiKangBingDu capsule, Influenza A (H1N1), Network pharmacology, Systems biology.

\begin{abstract}
Pharmacological mechanisms of Chinese herbal prescription are complicate since it contains heterogeneous components. These mechanisms are hardly understood by traditional methodology in the mass. Here we use network pharmacology approaches to explore mechanisms of JinChaiKangBingDu Capsule (JCKBD) against influenza A (H1N1) virus infection at a system level. Firstly, two web-based text mining systems were used for collecting the genes/proteins that associate with $\mathrm{H} 1 \mathrm{~N} 1$ virus infection and pharmacology process of ingredients in JCKBD from literature. The 1087 genes/proteins taken part in $\mathrm{H} 1 \mathrm{~N} 1$ infection course and 140 genes/proteins influenced by ingredients in JCKBD were found, which were demonstrated by traditional experiment. Secondly, H1N1 network and JCKBD network was constructed by using data of these genes/proteins interaction from multiple protein-protein interaction databases, respectively. Then, mapping JCKBD network to H1N1 network, produced subnetworks, considered the pharmacological networks of JCKBD against H1N1 virus infection. Thirdly, analysis based on topological structure of H1N1 network revealed the proteins with high central value in these pharmacological networks of JCKBD, meaning that these proteins play important roles in the course of JCKBD against H1N1 virus infection, such as TP53, CASP3, HSP90AA1, AKT1, RELA, NFKB1, MAPK1, etc. Finally, gene ontology (GO) enrichment of the proteins in the pharmacological networks shown that significant terms involved in cellular response to stress, apoptosis and signal transduction. Therefore, it was suggested that core mechanisms of JCKBD against H1N1 virus infection at a system level should be effects on signal transduction implicating in stress response and apoptosis.
\end{abstract}

\section{Introduction}

Influenza outbreaks frequently these years, especially influenza A (H1N1), which is an epidemic and pandemic infection and causes tremendous social impacts in addition to generating serious threats to the health and lives of global population [1]. While the Oseltamivir and other antiviral drugs were widely used in the treatment [2], but the drug resistance and potential limitations restrict their use [3]. To reduce such threats and impacts, governments have implemented or promoted anti-viral drugs [4]. How to find more potential effective drugs is the responsibility not for modern, but for Chinese 
medicine $(\mathrm{CM})$ or other medicines. $\mathrm{CM}$ has been used in the treatment of flu for many years and has been proved to be effective $[5,6]$. However, although many CM preparations show effectiveness in clinical treatment, the mechanisms haven't been explained for the reason of complexity and uncertainty, which needed more researches using different methods and techniques to explore. With more researches have been done on the CM compounds or monomers, network pharmacology approaches might be one of the efficient ways to explore the mechanisms of CM preparation, especially for prescription composed of multiple herbs. We can use the current biological data of diseases and compounds or monomers that included in the prescription to explore the possible mechanism of it. Then make related experiments to identify the results.

JinChaiKangBingDu capsule (JCKBD) is a new kind of CM preparation as an anti-influenza drug approved by Chinese government, consisting of Lonicera japonica, Forsythia suspense, Scutellaria baicalensis, Bupleurum chinense, Pinellia ternate, Rhizoma cyrtomii fortunei and Panax quinquefolius. It shows obvious anti-influenza virus effects in animal experiments and in vitro test [7], but the mechanisms against influenza are not enough for the reason of composition. Procession of interaction between virus and host to result in disease, as well as the mechanism of anti-viral drugs, is complex. System biology approaches can lead to a deeper understanding of complex systems as a whole [8]. Here, basing on the existing data of influenza onset, anti-influenza virus effect of drugs and the relationship between them, we explore the pharmacological mechanisms of JCKBD against H1N1 virus infections by network pharmacology approaches to find more evidences or clues in treatment or research of against influenza with JCKBD.

\section{Methods}

\section{Data Mining for Collecting Associated Genes/Proteins}

Genes/proteins associated with H1N1 were generated using PolySearch, a web-based text mining system for extracting relationships between human diseases, genes, mutations, drugs and metabolites [9]. In this study, select search restraints from the pull-down menus (Given drug, Find genes/proteins), input drug keyword "H1N1" and select default filter words and database (PubMed), no limit abstract and time. Minimum number of citations/references per gene/protein is 3. Marked sentences from database were artificially read so as to get rid of non-confidence genes/proteins.

Genes/proteins affected by JCKBD were available from PubChem. PubChem is organized as three linked databases within the NCBI's Entrez information retrieval system, providing information on the biological activities of small molecules [10]. Names of 60 major compounds in herbs of JCKBD were used as keywords to search in PubChem, including Phillyrin, forsythoside, ephedrine, pseudoephedrine, amygdalin, parietic acid, filicin, anesole, et al. Genes/proteins associated with the compounds were found from PubChem, and were regarded as genes/proteins affected by JCKBD in certain condition.

\section{Network Restructure with Protein-Protein Interaction}

Information on human protein-protein interactions with genes/proteins affected by JCKBD in certain condition or associated with H1N1 was obtained from databases, including BIND (Biomolecular Interaction Network Database), BioGRID (The General Repository for Interaction Datasets), DIP(Database of Interacting Proteins), HPRD 
(Human Protein Reference Database), IntAct(Database system and analysis tools for protein interaction data) and MINT (Molecular Interactions Database). This information was complimented with curated relationships parsed from the literature using Agilent Literature Search [11]. These datasets are mostly based on experimental evidence. This study did not include data that were deemed to be of lower quality. Networks of JCKBD and H1N1 were set up by the information, respectively. The protein-protein interaction networks were visualized using Cytoscape software [12].

\section{Network Centrality Analysis}

To define the importance of the proteins in H1N1, the network centrality analysis was performed in H1N1 network. In this study, the importance of a node (protein) was defined by degree centrality, which was defined as the number of edges incident to the node (i.e., the number of neighbors that a node has) [13]. It assesses the extent of influence that a node has on the network. The more neighbors a node has, the more important it is [14].

\section{Mapping between the Networks}

Mapping between the networks was conducted by using graph merge tool, which can find the union, intersection and difference of networks based on node identifiers/IDs. The nodes were matched according to ID mappings, i.e. the nodes whose IDs were mapped to each other were considered as the same node when the value of their selected attribute(s) match, and then merge the attribute of source networks into attributes in the resulting network. By this tool, JCKBD network mapped onto H1N1 network, produced new networks representing the groups of interacting proteins displaying critical effects of JCKBD in H1N1.

\section{Gene Ontology Analysis}

Gene ontology (GO) is a commonly used gene functional categorization system. To identify the function of networks, GO clustering analysis was performed with the proteins in the networks. For this purpose, the Biological Network Gene Ontology (BiNGO) tool [15] was used to statistically evaluate groups of proteins with respect to the existing annotations of the Gene Ontology Consortium (GOC). The degree of functional enrichment for a given cluster was quantitatively assessed ( $\mathrm{P}$ value) by hypergeometric distribution, implemented in BiNGO tool.

\section{Results}

\section{Influenza A (H1N1) network}

With text mining, 1087 genes/proteins associated with H1N1 were found from literature. A human protein interactome dataset among these genes/proteins was compiled from public databases and literature. This network was visualized as H1N1 network, including 691 nodes (proteins) and 2516 edges (interactions). In the protein network, the higher centrality value of a node is, the more significant it is. Centrality subnetwork was achieved by extracting the most relevant nodes which had all centrality values over the average, including 111 nodes and 668 edges (Figure 1), which represented a group of interacting proteins displaying a critical role in H1N1 pathogenesis. 


\section{JCKBD Network}

In certain condition 140 genes/proteins affected by JCKBD were found from PubChem.

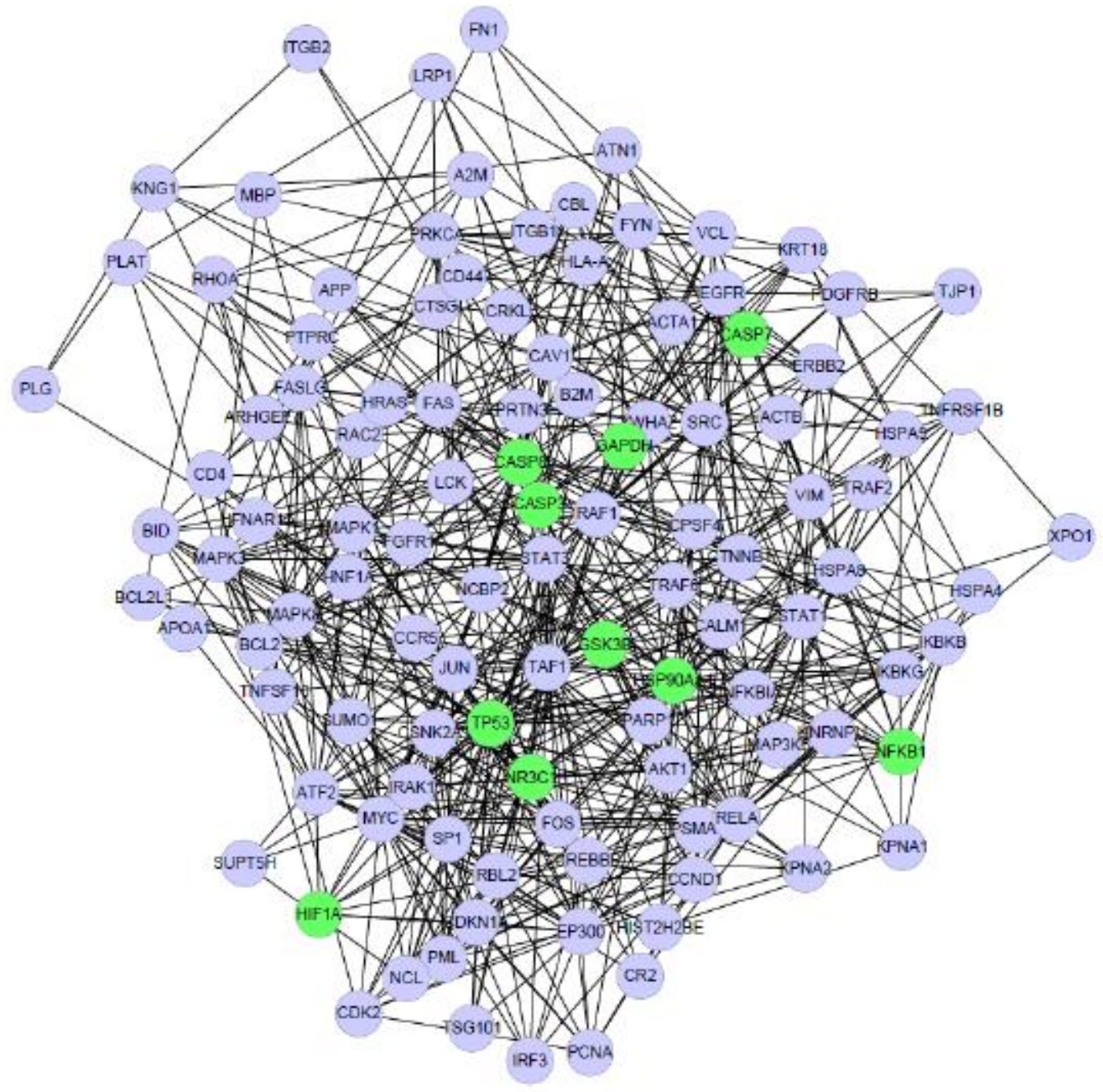

Figure 1. Centrality subnetwork of Influenza A (H1N1)

A human protein interactome dataset among these genes/proteins was compiled, and was visualized as JCKBD network, including 691 nodes (proteins) and 2516 edges (interactions) (Figure 2), which represented a group of interacting proteins displaying critical effects in pharmacological mechanism of JCKBD. By GO analysis, the network involved in cellular response to stress, regulation of programmed cell death, signal transduction (Table 1).

\section{Networks Relative to JCKBD Against Influenza A (H1N1)}

JCKBD network mapped onto H1N1 network produced two networks, representing two groups of interacting proteins displaying critical effects of JCKBD in treating H1N1. In the two networks, size of node was visualized according to centrality value of the node, which shown the proteins played important roles in JCKBD against H1N1, including TP53, NFKB1, CASP3, CASP8, GSK3B, NR3C1, CASP7, CASP9 (Figure $3)$. 


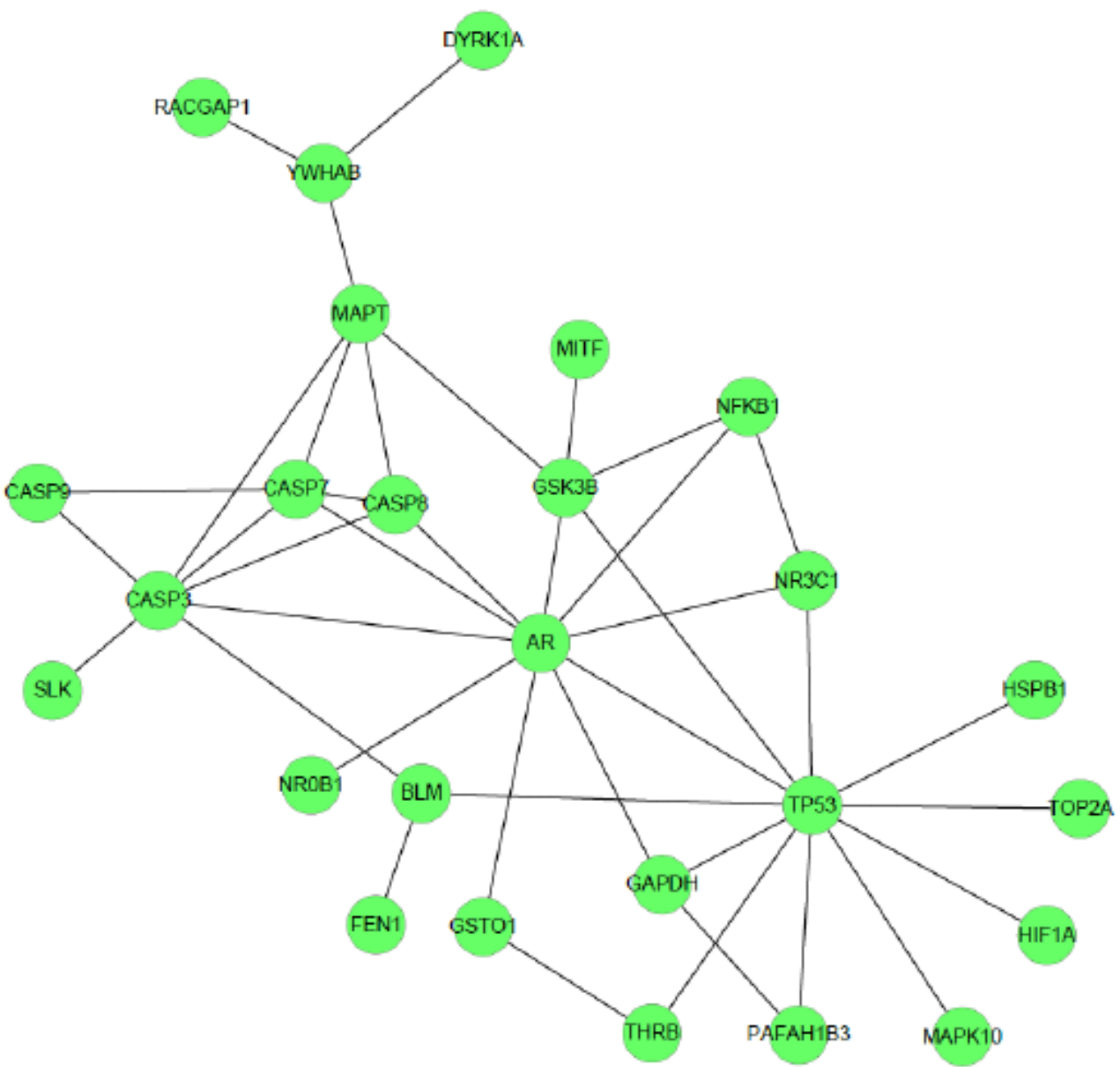

Figure 2. JCKBD network

Table 1. Biological categories of JCKBD network

\begin{tabular}{lll}
\hline ID & Description & $P$-val \\
\hline 33554 & Cellular response to stress & $4.16 \mathrm{E}-07$ \\
43067 & Regulation of programmed cell death & $9.68 \mathrm{E}-07$ \\
7165 & Signal transduction & $1.28 \mathrm{E}-05$ \\
\hline
\end{tabular}

Figure 3. Networks relative to JCKBD against Influenza A (H1N1) 


\section{Discussions}

Virus want the host cell to provide most of substance, energy and environment for what its life cycle needs, so must cause the host cell to make adjustments that facilitate its efficient replication, after entering the body. In contrast, the host cell makes response against the virus. Over response to virus, disease is triggered [16].

H1N1 virus can cause the host cell to support its efficient duplication by activating intracellular signaling. NF- $\kappa \mathrm{B}$ (NFKB1) signaling pathway was activated by proteins of H1N1 virus, such as HA, NA, M, NP and NS [16, 17]. Expression of NF- $\kappa B-d e p e n d e n t$ TRAIL and Fas/FasL were required for efficient replication of influenza virus and appropriate activation of NF- $\kappa \mathrm{B}$ was prerequisite for infection of the virus $[16,17]$. Synthesis of virus RNA was regulated by NF- $\kappa B$ signaling pathway. Knockout of p65, a member of NF- $\kappa B$ family, decreased replication and synthesis of the virus RNA [16, 17].

Recent studies have demonstrated that H1N1 virus infection activates the phosphatidylinositol 3-kinase (PI3K)/Akt by binding of influenza NS1 protein to the p85 regulatory subunit of PI3K and induce activation of downstream signal molecules, such as NF- $\mathrm{B}$, p53, GSK3B, CASP8, CASP9, inhibiting virus induced apoptosis is beneficial for virus replication [18-21]. The NS1 dramatically weakened the interaction between Apaf-1 and Hsp90 but enhanced its interaction with cytochrome c (Cyt c), promoting the Apaf-1 to associate with Cyt $\mathrm{c}$ and thus facilitate the activation of CASP9 and CASP3/7 [22]. Inhibition of NF- $\kappa$ B, GSK3B, TP53, CASP3, CASP7, CASP8 and CASP9 reduced replication of the virus [19, 22-24].

JCKBD is a new kind of anti-viral compound Chinese Medicinal preparation. In this study, network analysis showed that the possible pharmacological mechanisms of JCKBD against $\mathrm{H} 1 \mathrm{~N} 1$ virus infection could be regulating programmed cell death and signal transduction, involved NF- $\mathrm{B}$, GSK3B, TP53, CASP3, CASP7, CASP8 and CASP9. Therefore, it was suggested that JCKBD could affect the function of intracellular signaling to regulate apoptosis and reduce replication of H1N1 virus.

Although in this study the suggestion was presented by the network approach, the genes/proteins affected by JCKBD or the genes link to H1N1 were not completely obtained by large-scale and high-throughput text mining, and information on human protein-protein interactions was yet incomplete so far. Thus, reconstruction of JCKBD network corresponding to $\mathrm{H} 1 \mathrm{~N} 1 \mathrm{will}$ need to be further gone into. The network approach did not identify whether or not the interaction effect was synergistic or antagonistic. This quantitative analysis of interaction effect in combination will be accomplished by drug network integrated with quantitative data in the future.

\section{Summary}

The possible mechanisms of JCKBD against influenza A (H1N1) virus could be involved in the function of intracellular signaling and regulating apoptosis to reduce replication of influenza $\mathrm{A}(\mathrm{H} 1 \mathrm{~N} 1)$ virus.

\section{Acknowledgement}

This research is supported by the projects from the National Natural Science Foundation of China (Grant No. 81102872). 


\section{References}

[1] N.C.Y. Yeung, J.T.F. Lau, K.C. Choi, S. Griffiths, Population Responses during the Pandemic Phase of the Influenza A(H1N1)pdm09 Epidemic, Hong Kong, China, Emerging infectious diseases, 23 (2017) 813-815.

[2] T.M. Lopes e Souza, N. Fintelman-Rodrigues, P.C. Resende, M. Mesquita, T.S. Gregianini, F.A. Bozza, A.C. Pecego, S.B. Fernandes, A.L. Cury, I.N. Riediger, M.M. Siqueira, Oseltamivir-resistant influenza A(H1N1)pdm2009 strains found in Brazil are endowed with permissive mutations, which compensate the loss of fitness imposed by antiviral resistance, Memorias do Instituto Oswaldo Cruz, 110 (2015) 101-105.

[3] D. Tamura, R.L. DeBiasi, M. Okomo-Adhiambo, V.P. Mishin, A.P. Campbell, B. Loechelt, B.L. Wiedermann, A.M. Fry, L.V. Gubareva, Emergence of Multidrug-Resistant Influenza A(H1N1)pdm09 Virus Variants in an Immunocompromised Child Treated With Oseltamivir and Zanamivir, The Journal of infectious diseases, 212 (2015) 1209-1213.

[4] A. Kamradt-Scott, The politics of medicine and the global governance of pandemic influenza, International journal of health services : planning, administration, evaluation, 43 (2013) 105-121.

[5] R.K. Ganjhu, P.P. Mudgal, H. Maity, D. Dowarha, S. Devadiga, S. Nag, G. Arunkumar, Herbal plants and plant preparations as remedial approach for viral diseases, Virusdisease, 26 (2015) 225-236.

[6] M.R. Talactac, M.Y. Chowdhury, M.E. Park, P. Weeratunga, T.H. Kim, W.K. Cho, C.J. Kim, J.Y. Ma, J.S. Lee, Antiviral Effects of Novel Herbal Medicine KIOM-C, on Diverse Viruses, PloS one, 10 (2015) e0125357.

[7] J. Zhong, X. Cui, Y. Shi, Y. Gao, H. Cao, Antiviral activity of Jinchai capsule against influenza virus, Journal of traditional Chinese medicine $=$ Chung $\mathrm{i}$ tsa chih ying wen pan, 33 (2013) 200-204.

[8] I. Muqbil, G.W. Bao, R. El-Kharraj, M. Shah, R.M. Mohammad, F.H. Sarkar, A.S. Azmi, Systems and Network Pharmacology Approaches to Cancer Stem Cells Research and Therapy, Journal of stem cell research \& therapy, Suppl 7 (2012).

[9] D. Cheng, C. Knox, N. Young, P. Stothard, S. Damaraju, D.S. Wishart, PolySearch: a web-based text mining system for extracting relationships between human diseases, genes, mutations, drugs and metabolites, Nucleic acids research, 36 (2008) W399-405.

[10]S. Kim, P.A. Thiessen, E.E. Bolton, J. Chen, G. Fu, A. Gindulyte, L. Han, J. He, S. He, B.A. Shoemaker, J. Wang, B. Yu, J. Zhang, S.H. Bryant, PubChem Substance and Compound databases, Nucleic acids research, 44 (2016) D1202-1213.

[11]A. Vailaya, P. Bluvas, R. Kincaid, A. Kuchinsky, M. Creech, A. Adler, An architecture for biological information extraction and representation, Bioinformatics, 21 (2005) 430-438.

[12] P. Shannon, A. Markiel, O. Ozier, N.S. Baliga, J.T. Wang, D. Ramage, N. Amin, B. Schwikowski, T. Ideker, Cytoscape: a software environment for integrated models of biomolecular interaction networks, Genome research, 13 (2003) 2498-2504.

[13] K. Park, D. Kim, Localized network centrality and essentiality in the yeast-protein interaction network, Proteomics, 9 (2009) 5143-5154. 
[14]H. Jeong, S.P. Mason, A.L. Barabasi, Z.N. Oltvai, Lethality and centrality in protein networks, Nature, 411 (2001) 41-42.

[15]S. Maere, K. Heymans, M. Kuiper, BiNGO: a Cytoscape plugin to assess overrepresentation of gene ontology categories in biological networks, Bioinformatics, 21 (2005) 3448-3449.

[16] J. Peng, J. Jiao, J. Li, W. Wang, Q. Gu, T. Zhu, D. Li, Pyronepolyene C-glucosides with NF-kappaB inhibitory and anti-influenza A viral (H1N1) activities from the sponge-associated fungus Epicoccum sp. JJY40, Bioorganic \& medicinal chemistry letters, 22 (2012) 3188-3190.

[17]J.R. Tisoncik, R. Billharz, S. Burmakina, S.E. Belisle, S.C. Proll, M.J. Korth, A. Garcia-Sastre, M.G. Katze, The NS1 protein of influenza A virus suppresses interferon-regulated activation of antigen-presentation and immune-proteasome pathways, The Journal of general virology, 92 (2011) 2093-2104.

[18] Y.K. Shin, Y. Li, Q. Liu, D.H. Anderson, L.A. Babiuk, Y. Zhou, SH3 binding motif 1 in influenza A virus NS1 protein is essential for PI3K/Akt signaling pathway activation, Journal of virology, 81 (2007) 12730-12739.

[19]X. Wang, Y. Shen, Y. Qiu, Z. Shi, D. Shao, P. Chen, G. Tong, Z. Ma, The non-structural (NS1) protein of influenza A virus associates with p53 and inhibits p53-mediated transcriptional activity and apoptosis, Biochemical and biophysical research communications, 395 (2010) 141-145.

[20]X. Lu, A. Masic, Q. Liu, Y. Zhou, Regulation of influenza A virus induced CXCL-10 gene expression requires PI3K/Akt pathway and IRF3 transcription factor, Molecular immunology, 48 (2011) 1417-1423.

[21]J.L. Murray, N.J. McDonald, J. Sheng, M.W. Shaw, T.W. Hodge, D.H. Rubin, W.A. O'Brien, D.F. Smee, Inhibition of influenza A virus replication by antagonism of a PI3K-AKT-mTOR pathway member identified by gene-trap insertional mutagenesis, Antiviral chemistry \& chemotherapy, 22 (2012) 205-215.

[22]C. Zhang, Y. Yang, X. Zhou, Z. Yang, X. Liu, Z. Cao, H. Song, Y. He, P. Huang, The NS1 protein of influenza A virus interacts with heat shock protein Hsp90 in human alveolar basal epithelial cells: implication for virus-induced apoptosis, Virology journal, 8 (2011) 181.

[23]G. Chase, T. Deng, E. Fodor, B.W. Leung, D. Mayer, M. Schwemmle, G. Brownlee, Hsp90 inhibitors reduce influenza virus replication in cell culture, Virology, 377 (2008) 431-439.

[24]S. Pleschka, RNA viruses and the mitogenic Raf/MEK/ERK signal transduction cascade, Biological chemistry, 389 (2008) 1273-1282. 